\title{
CONDUCTAS DE AUTOCUIDADO Y MANIFESTACIONES PERIMENOPÁUSICAS EN MUJERES DE LA COMUNA DE CONCEPCIÓN, CHILE
}

\author{
SELFCARE BEHAVIOUR AND THE TYPICAL CHARACTERISTICS OF \\ WOMEN IN THE PERIM ENOPAUSIC PERIOD, CONCEPCIÓN, CHILE
}

\author{
ELIZABETH M. BASTÍAS ARRIAGADA* y OLIVIA SANHUEZA ALVARAD 0**
}

\begin{abstract}
RESUMEN
La presenteinvestigación tuvo como objetivo valorar las conductas deautocuidado y manifestaciones características dela mujer del período perimenopáusico, y su relación con el perfil bio demográfico y social de estas, entre los 45 y 54 años. Se trata de un estudio explicativo, transversal, correlacional. La muestra estuvo formada por 148 mujeres de la comuna de Concepción, Octava Región, Chile. Losinstrumentos recolectores de datosutilizadosfueron: Cuestionario elaborado por la autora de este estudio, sobre antecedentes generales de la mujer en periodo perimenopáusico, Cuestionario especifico de Calidad deVida en la menopausia, Universidad de Toronto adaptado por la Fundación PROSAM en Chileen 1997 y Cuestionario de autocuidado deVoda. El análisis de los datos fue a través de estadística descriptiva y prueba deANOVA. De acuerdo con los resultados de este estudio, el perfil bio demográfico y social de las mujeres no influenciaría la aparición de manifestaciones características del período, y las actividades de autocuidado generadas por las mujeres serían independientes deestos perfiles. Entrelas manifestaciones observadas, las vasomotoras no son significativas; de las manifestaciones psicológicas, lo más sobresaliente es la presencia de nerviosismo y ansiedad, como también sentirse deprimida o triste. Priman las manifestaciones físicas, principalmente presencia de flatulencia, gases o hinchazón abdominal, los dolores muscularesy/o articulares, cansancio, disminución de la fuerzay dela resistencia física como también cambiosen el deseo sexual y el evitar las relaciones sexuales. Las actividades de autocuidado que más se destacan, son las de promoción de bienestar, percibiéndose la necesidad de recibir mayor información respecto a este período de sus vidas, el cual es necesario enfrentarlo, desde un punto de vista sanitario y social con una programación acorde y la misma naturalidad con que se enfrentan otras etapas de la mujer, como la reproductiva, pero contando con el apoyo adecuado y necesario para su bienestar.
\end{abstract}

Palabras claves: Perimenopausia, conducta de autocuidado, manifestaciones del climaterio.

\begin{abstract}
The following research was conducted in order to assess the selfcare behavior and the typical characteristics of women in the perimenopausic period, and its relation to the biodemographic and social profile, being their ages among 45 to 54 . This is an explicative, cross sectional and correlational study. Sample was conformed by 148 women from Concepción County( 8o Region) Chile. Data collection tools were: a questionnaire done by the author of the present research, which includes the general information of the woman at perimenopausic stage, specified questionnaireabout Quality of Life at menopause, (Toronto University adapted by PROSAM foundation in Chile in 1997) and questionnaire about Selfcare life. Data analysis was done by descriptive statistics and Anova. According, the findings, the biodemographic and social profiles of women, would not influence the appearance of manifestation characteristic of this period, and the activities of self-care generated by women would be independent from these profiles. Among some manifestations that were observed, vasomotor ones, were not significant; whereas among the psychological ones, the stress and anxiety turned out to be the most outstanding as well as being depressed and sad. Physical manifestations, primarily flatulence, gas and abdominal inflation, muscle aches, fatigue, decrease of strength and physical resistance. Women also manifested changes in sexual behavior and avoid sexual relations. The activities of self-care which are most highlighted, are: well-being promotion, whereby women indicated the need to receive major information regarding their life's, period they were experiencing which is necessary to face, from a healthy and social point of view with a program according and as natural as to face other periods the women face, like the reproductive one, but counting on thenecessary support for their well-being.
\end{abstract}

Keywords: Perimenopausia, selfcare, manifestation of climaterio.

Recepcionado: 03.03.2004. Aceptado: 04.06.2004

*Enfermera, docente Universidad San Sebastián, campus Osorno, Chile. E-mail: ebastias@uss.cl

**Enfermera, docente Depto. de Enfermería, Universidad de Concepción, Chile. E-mail: osanhue@udec.cl 


\section{INTRODUCCIÓN}

Los cambios bio-demográficos de la población chilena, caracterizados por una mayor expectativa de vida, han producido el subsiguiente aumento dela población mayor. Ello ha incrementado el porcentaje demujeresque alcanzan la etapa del climaterio, lográndose un reconocimiento crecientedelosproblemas que aquejan a las mujeres en etapa perimenopáusica (Valdivia, 1993). Si partimos del supuesto de que la función reproductora femenina puede cesar entre los 45 y los 50 años, se ha estimado que, a partir del año 2000, una de cada 2 a 3 mujeres podrá esperar una vida postmenopausia entre 25 a 30 años (SERNAM , 1994). Es así como en Chile el Censo 2002 señala que en el país las mujeres entre 45 y 49 años es de 462.910 y entre 50 y 54 años es de 389.603 , proporción similar es la que se encuentra en la comuna de Concepción: las mujeres entre 45 y 54 años son 7.006 y las mujeres en el rango de 50 a 54 años es de 6.086.

Uno de los cambios fundamentales de la edad intermedia -la disminución de las capacidades reproductoras- afecta a hombres y mujeres de manera diferente. El evento biológico dela menopausia implica quela mujer deja de ovular y de menstruar y que ya no puede concebir hijos. Esto ocurre al rededor delos cincuenta años, en promedio, pero puede suceder muy normalmente varios años antes o después (Upjohn, in: Papalia, 1992). El tiempo de unos dos a cinco años durante los cuales, el cuerpo de la mujer experimenta los diversos cambios fisiológicos que conducen a la menopausia se conoce técnicamente como el período climatérico" (Papalia, 1992) y, más recientemente, como etapa perimenopáusica (Lavín, in: González y col., 1998).

Indudablementesu calidad devida se pue de ver amenazada y coincide con lo expresado en el "marco epidemiológico" del Programa de Salud de la Mujer, del Ministerio de Salud (1995), en el sentido de reconocer que las necesidades de salud de la mujer presentan una amplia variedad, según las condicio- nes de vida que enfrenten y las diferentes etapas desu ciclo vital, todo lo cual requiereidentificar condiciones específicas de riesgo, a fin de proponer estrategias adecuadas de abordaje.

Al respecto, los conceptos que actualmente se utilizan son los siguientes:

- La definición médica dela menopausia, asumida también por la Organización M undial dela Salud, se refierea"la cesación dela función ovárica, la cual resulta en una amenorrea permanente (ausencia de menstruación)". Ésta sucede dentro del período perimenopaúsico, clasificándose en natural 0 fisiológica, cuando sobreviene como resultado del cese espontáneo de la función ovárica, y en iatrogénica o artificial, cuando ha sido provocada por la extirpación de los ovarios o por un agente externo (radioterapia, quimioterapia, histerectomía) (Gerer, 1993).

- El período de la perimenopausia, Li y col. (2000) lo definen como "la transición alrededor dela menopausia de unos pocos años antes y hasta un año después del cese permanentedela menstruación", definición que se complementa con "el período de transición entre la menstruación normal y la finalización total de la misma", Merck (2001). Otra definición de perimenopausia señala que se caracteriza por una declinación progresiva dela función ovárica, causando trastornos en la ciclicidad y/o cantidad del sangrado uterino, así como manifestaciones de deficiencia estrogénica y elevación de las gonadotrofinas (Lavín, in: González y col., 1998). Los conceptos de perimenopausia y climaterio son definidos indistintamente por varios autores.

Las manifestaciones asociadas con la perimenopausia se podrían agrupar en:

- M anifestaciones vasomotoras: como sofocos, bochornos y sudores nocturnos.

- M anifestaciones psicológicas: según la literatura se ha observado gran diversidad desín- 
tomaspsicológicos. Así, no está claro en qué medida estas "descripciones sintomáticas" hacen referencia a algún factor general de bienestar o desánimo 0 a situaciones psicológicas más precisas, como depresión, fatiga 0 irritabilidad.

- Manifestacionesfísicas: tales como disminución de la fuerza física, presencia de signos deatrofia, incontinencia deorina al realizar ciertos esfuerzos, como puede ser al toser, estornudar o reír, insomnio, cansancio, perdida dela memoria, dificultades deconcentración, dolores de cabeza.

- M anifestacionesasociadasa la sexualidad: las mujeres que asisten a las consultas médicas a causa de trastornos perimenopáusicos 0 postmenopáusicos suelen quejarse de sequedad vaginal y la consiguiente dispareunia, también una disminución del interés y del placer sexual.

Por otro lado, la fundamentación teórica de esta investigación está centrada en la teoría del autocuidado, que abarca las necesidades de autocuidado universales de todos los individuos, así como también las necesidades específicas del desarrollo físico, social y psicológico. También incluye la situación de enfermedad o lesión, y cómo ésta se relaciona con los profesionales del cuidado de la salud, aspecto relevantesi consideramos quelas mujeres, como cualquier individuo, no seencuentran aisladas en su entorno. Orem (1991) enfatiza que la realización del autocuidado requiere la acción intencionada y calculada, que está condicionada por el conocimiento y repertorio de habilidades de un individuo, y se basa en la premisa que los individuos saben cuando necesitan ayuda y, por lo tanto, son conscientes de las acciones específicas que necesitan realizar. Sin embargo, pueden escoger entre distintas opciones en sus conductas de autocuidado. $Y$ en al gunas circunstancias, un individuo puede decidir, por cualquier razón, no iniciar la conducta de autocuidado cuando es necesaria.

El autocuidado podría considerarse como la capacidad de un individuo para realizar todas las actividades necesarias para vivir y sobrevivir. Orem (1993) considera el concepto "auto" como la totalidad de un individuo, incluyendo no sólo sus necesidades físicas, sino también las psicológicas y espirituales, y el concepto "cuidado" como la totalidad de actividadesqueun individuo inicia paramantener la vida y desarrollarse de una forma que sea normal para él. Así, el autocuidado es la práctica de actividades quelos individuos inician y realizan en su propio beneficio para el mantenimiento de la vida, la salud y el bienestar (Orem, 1993).

Esta teoría se encuentra inserta dentro de un modelo conceptual, cuya autora es Dorotea Orem (1993), y el cual integra tres teorías relacionadas: la teoría del autocuidado (que es la que servirá de marco para este estudio); la teoría del déficit del autocuidado y la teoría de los sistemas de enfermería.

En la Figura 1 se encuentra representada la persona sana, en una delicada balanza, capaz de satisfacer los requi sitos de autocuidado universal, con las capacidades de autocuidado que ha adquirido a lo largo de su historia, y que lleva a mantener un autocuidado eficaz que se traduce en un nivel óptimo de bienestar. Cuando estas capacidades no logran mantener el delicado equilibrio comienza a ser necesaria la acción de enfermería a fin de restablecer el equilibrio, encontrándonos así en una situación de autocuidado ineficaz que requierela acción deagentes desalud queayuden a restablecer el bienestar de la mujer. Las capacidades de autocuidado están directamente influenciadas por la cultura, el grupo social en el que está inserta la mujer, el conocimiento de habilidades de autocuidado y el repertorio para mantenerlas, asimismo como dela capacidad para hacer frente a las dificultades con las que se encuentrea lo largo desu historia.

Este período en nuestro medio ha sido estudiado principalmente por Blümel y col., desde un punto de vista biológico principalmente. Blümel y col. (1991) expresan que el 


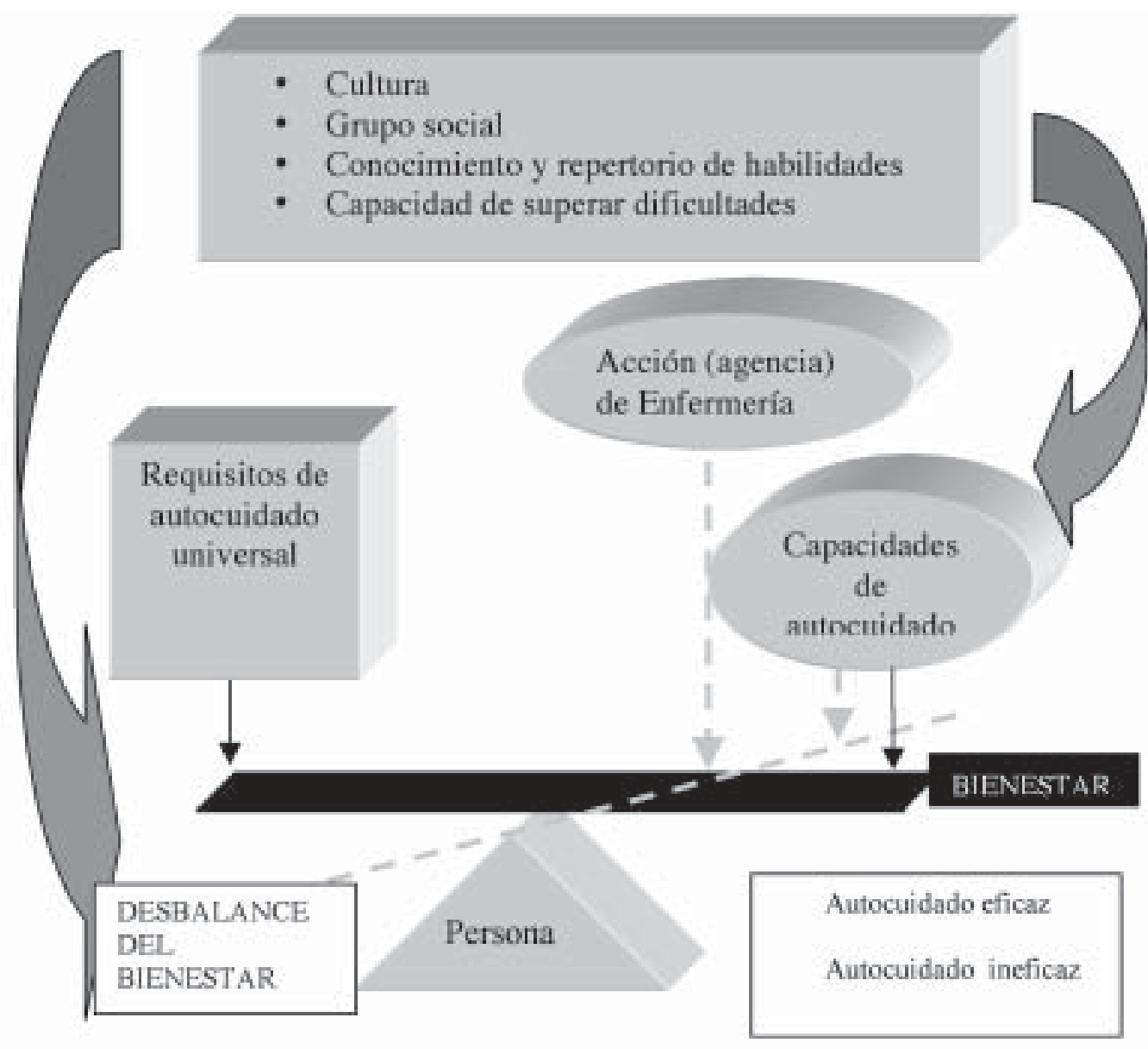

Figura 1. M odelo ilustrativo presentado por las autoras para representar el fenómeno en estudio.

Fuente: Investigación “Conductas de autocuidado y manifestaciones de la mujer en su etapa perimenopausica, comuna de Concepción, 2003”.

cese de la función ovárica se expresa objetivamente con el término de la menstruación, lo cual seacompaña de una serie de síntomas deintensidad variable, queafectarían el desarrollo normal de la vida de la mujer. Este primer estudio señala los bochornos ( sensación de calor ascendente) como comprometiendo a dos tercios de las mujeres, pudiendo comenzar junto con los primeros trastornos del ciclo y prolongarse durante años después del término de las menstruaciones. Otro signo, la cefalea, queja frecuente, puede llegar a ser invalidante. Otros síntomas altamente frecuentes son depresión, ansiedad e insomnio. Los problemas sexuales, seseñala, son casi una constante. La pérdida dela autoestima es dra- mática, puesto que consideran quese trata de mujeres jóvenes aún, muchas de ellas en una etapa de plena actividad y realización. Blümel (1998) evaluó el efecto del climaterio en la calidad de vida en 481 mujeres peri y postmenopáusicas en Santiago (Chile), concluyendo que el climaterio produce pérdida de la calidad de vida de la mujer, en que las mujeres peri o postmenopáusicas tendrían peor calidad de vida que las mujeres con ciclos normales, existiendo mayores molestias vasomotoras, mayor deterioro psicosocial, mayor trastorno físico y mayores trastornos de sexualidad. Esteestudio fueposterior al realizado por Blümel, Blakey Etchevers (1996), donde estudiaron el ausentismo por causas médicas de 444 
mujeres de 40 años o más, trabajadoras deun hospital público en Santiago de Chile, en que identificaron que las molestias vasomotoras y la caída de la sexualidad predominan en el período postmenopáusico, mientras que la depresión y la pérdida de la capacidad laboral lo hacen en el período perimenopáusico.

A nivel regional, Salazar (2003) señala entre los resultados de su trabajo de investigación realizado en mujeres climatéricas trabajadoras dedosinstituciones públicas chilenas, queel $92,2 \%$ presentó déficit parcial de autocuidado respecto al climaterio, identificando algunas acciones como las más frecuentemente utilizadas para el alivio de los síntomas en esta etapa. No encuentra asociación de deterioro en el desempeño laboral en las participantes del estudio.

\section{HIPÓTESISY OBJETIVOS}

\section{H ipótesis general}

Las mujeresen período perimenopáusico realizan conductas de autocuidado relacionadas directamente con las manifestaciones de ese período, y éstas se van incrementando en la medida en que se presentan en mayor número e intensidad en esta etapa.

\section{Propósito}

Conocer las conductas de autocuidado y manifestaciones características de la mujer en el período perimenopáusico, y su relación con el perfil bio-demográfico y social de éstas.

\section{O bjetivos específicos}

- Identificar perfil bio-sociodemográfico de las mujeres en etapa perimenopáusica de la comuna de Concepción, Chile.

- Identificar los patrones de autocuidado de las mujeres en etapa perimenopáusica de la comuna de Concepción, Chile.

- Identificar manifestaciones representativas de la etapa de perimenopausia de las mujeres de la comuna de Concepción, Chile.

- Determinar la intensidad de las manifestaciones perimenopáusicas de las mujeres de la comuna de Concepción, Chile.

- Relacionar el perfil bio socio-demográfico delas mujeres en etapa perimenopáusica de la comuna de Concepción, Chile, con conducta deautocuidado y las manifestaciones que aparecen durante este período.

\section{MATERIALESY MÉTODOS}

Diseño detipo explicativo, transversal, correlacional (Hernández, Fernández, Baptista, 1998). La unidad de análisis de esta investigación está constituida por la mujer entre 45 y 54 años de la comuna de Concepción.

\section{Instrumentos recolectores de datos}

- Cuestionario elaborado por los autores de esteestudio, sobreantecedentes generales de la mujer en período perimenopaúsico. Este cuestionario consta de preguntas relacionadas con características bio-sociodemográficas de las mujeres como edad, previsión, escolaridad, pareja, pertenencia a grupos religioso-comunitarios, asistencia a controles de salud, así como preguntas sobre características ambientales (tipo devivienda, $\mathrm{n}$ - de hijos que viven con ella, ingresos económicos).

- Cuestionario deautocuidado deVoda, creado en 1984 y dirigido a mujeres en período perimenopaúsico (validado por Mamede, 1988, Brasil). En nuestro país fue presentado por la revista EPAS de la Pontifica Universidad Católica, sin que consten registros dehaberseutilizado en alguna investigación en el ámbito nacional. Utiliza escala tipo Likert con 40 ítemes.

- Cuestionario específico de Calidad deVida en la menopausia, Universidad de Toronto, presentado por Hilditch y cols., 1996. Este 
instrumento fue propuesto como cuestionario específico para evaluar calidad devida en la menopausia, basándoseen la experiencia reportada por mujeres que atraviesan esta etapa. Fue adaptado por la Fundación PROSAM en Chile en 1997 y validado por Blümel y col. (1998). Utiliza escala tipo Likert, y mide en forma independiente las manifestaciones que pudieran presentarse en el período perimenopaúsico de la mujer en cuatro dominios: vasomotor, psicosocial, físico y sexual, lo queen total suma 29 ítems.

\section{Población en estudio}

- El universo de este estudio corresponde a 12.621 (INE, 2000) mujeres, quese encuentran entre los 45 y 54 años y que residen en la comuna de Concepción. La muestra obtenida al azar correspondió a 148 mujeres del listado del Registro Electoral de la comuna de Concepción, coincidente con el INE.

- La unidad de análisis de esta investigación está constituida por la mujer entre 45 y 54 años de la comuna de Concepción.

\section{Procesamiento de los datos}

Los datos fueron codificados e incorporados en una base de datos en programa SPSS, versión 8.0 (paquete estadístico para ciencias sociales). Posteriormente se analizaron con este programa estadístico.

Estadística descriptiva: Para la obtención del perfil de las características de la muestra.

Estadística inferencial: Para conocer la relación de las variables independiente con la dependiente se utilizan pruebas estadísticas paramétricas (Hernández y col., 1994). Se utilizó AN OVA para anal izar las relaciones entre variables. Dentro deesta prueba también serealizan comparaciones a posteriori, a fin deana- lizar hacia que grupo es favorable la diferencia (mínima diferencia significativa de Tukey). Para poder analizar además las diferencias entre variables se utilizó el eta cuadrado que da información acerca de la intensidad de la relación entre variables; este eta cuadrado es un coeficiente de determinación análoga al $R$ cuadrado en la regresión.

Prueba piloto: Esta prueba se aplicó a 30 mujeres, con la finalidad de determinar el grado decomprensión delosinstrumentosanteriormentemencionados.

Como no se presentaron dificultades de comprensión durantesu aplicación, estos datos recopilados para la prueba piloto pasaron a formar parte de la muestra.

\section{Validez}

Cuestionario específico de Calidad de Vida en la M enopausia

Estecuestionario, que mide en forma separadalas manifestaciones que se pueden presentar en la etapa cercana a la menopausia, originalmente fue validado por sus autores con instrumentos psicométricos y clínicos. Con posterioridad, fuetraducido al español por un comité de expertos y, para garantizar su adecuada traducción, fuetraducido nuevamente al inglés por un nativo de esta lengua y, asimismo, esta nueva versión de traducción fue llevada al español.

Lafundación PROSAM en Chilevalida este instrumento en 1997 con mujeres consultantes, no encontrándose falla en la comprensión ni interpretación de las preguntas.

Blümel y cols., en 1998, validaron esteinstrumento en el trabajo "Efecto del climaterio en la Calidad deVida".

Cuestionario de Autocuidado de Voda (1984), dirigido a mujeres en período perimenopáusico (validado por M amede, 1988, Brasil). En nuestro país ha sido presentado por la revista 
EPAS dela Pontifica Universidad Católica, en el año 1988.

\section{Confiabilidad}

Cuestionario específico de Calidad deVida en la M enopausia

En el presente trabajo las confiabilidadesfueron las siguientes:

Dominio vasomotor: Alfa 0.79

Dominio psicosocial: Alfa 0.79

Dominio físico: Alfa 0.85

Dominio sexual: Alfa 0.73

$\mathrm{N}=148$

Para obtener estos valores de al fa deCronbach, los datos del cuestionario se agruparon en índices, representados por los dominios, que son los que se expusieron:

En el estudio original deH ilditch y cols., 1996:

Dominio vasomotor: Alfa 0.82

Dominio psicosocial: Alfa 0.81

Dominio físico: Alfa 0.87

Dominio sexual: Alfa 0.89

$\mathrm{N}=144$

En el estudio realizado en Chilepor PROSAM :

Dominio vasomotor: Alfa 0.81

Dominio Psicosocial: Alfa 0.83

Dominio físico: Alfa 0.89

Dominio sexual: Alfa 0.86

$\mathrm{N}=481$

\section{Cuestionario de Autocuidado de Voda}

Actividades de apoyo del funcionamiento normal: 0.49

Actividades de prevención o control de los procesos de enfermedad o lesión: 0.47

Actividades de promoción de bienestar: 0.67
Dentro de estas actividades, los valores de alfa de Cronbach son pequeños para lo que se esperaría para un instrumento adecuado para ser aplicado, pero en estas actividades están incluidas tanto las positivas como las negativas que ayudarían o entorpecerían (en cada caso) al desarrollo de esta etapa en la mujer. Por esta razón, para obtener los resultados de esta investigación, se procedió a dar valores positivos a dichas actividades con la finalidad de que la escala reflejara lo deseado.

\section{RESULTADOS}

En la Tabla 1 seaprecia que la mayor parte de las mujeres de la muestra se concentra en el grupo demujeres menores de 50 años correspondientesa un $54,1 \%$, dato quecoincidecon los del Censo 2002, recogidos en la comuna deConcepción, Octava Región como también a nivel nacional, en una proporción similar (INE, 2003).

Respecto a su nivel educacional, un 52,7\% deellas refiere haber completado nivel deenseñanza media, teniendo un 31,3\% cursado estudios superiores completos, lo cual representa un porcentaje que no estaba presente en estudios anteriores. Estos datos contrastan en parte con los recogidos en el Censo 2002, quemuestran quela mayor parte de la población femenina, tanto a nivel regional como a nivel nacional, ha completado principalmente la educación media en cualquiera de sus variantes (media común, humanidades, media comercial, media industrial, media agrícola, media marítima o normal), siendo pocas las mujeres que llegan a cursar estudios universitarios, no superando el $13 \%$ tanto parala Octava Región como para el país (INE, 2003).

Respecto del estado conyugal, un $62,8 \%$ de las mujeres encuestadas refiere tener pareja. Si comparamos este dato con el Censo 2002 (INE, 2003), nos encontramos que en éste existeuna proporción aún mayor de mujeres que refieren tener pareja. 
Tabla 1. Características bio socio demográficas de las mujeres en etapa perimenopáusica. Comuna de Concepción. 2002.

\begin{tabular}{|c|c|c|}
\hline Variable & $\mathrm{n}$ & $\%$ \\
\hline \multicolumn{3}{|l|}{ Edad } \\
\hline 45-49 años & 80 & 54.1 \\
\hline 50-54 años & 68 & 45,9 \\
\hline \multicolumn{3}{|l|}{ Nivel educacional } \\
\hline Básica & 24 & 16,2 \\
\hline Media & 78 & 52,7 \\
\hline Universitaria & 46 & 31,1 \\
\hline \multicolumn{3}{|l|}{ Estado conyugal } \\
\hline Con pareja & 93 & 62,8 \\
\hline Sin pareja & 55 & 37,2 \\
\hline \multicolumn{3}{|l|}{ Hiios en el hogar } \\
\hline Con hijos en el hogar & 125 & 84,5 \\
\hline Sin hijos en el hogar & 23 & 15,5 \\
\hline \multicolumn{3}{|l|}{ Trabajo } \\
\hline Trabajo remunerado & 79 & 53,4 \\
\hline Trabajo del hogar & 69 & 46,6 \\
\hline \multicolumn{3}{|l|}{ Orientación religiosa } \\
\hline Evangélica & 21 & 14,2 \\
\hline Católica & 108 & 73 \\
\hline Otros (Testigo de Jehová, M ormón) & 3 & 2,0 \\
\hline No tiene & 16 & 10,8 \\
\hline \multicolumn{3}{|l|}{ Pertenencia a grupo comunitario } \\
\hline $\mathrm{SI}$ & 20 & 13,5 \\
\hline NO & 128 & 86,5 \\
\hline \multicolumn{3}{|l|}{ Previsión de salud } \\
\hline ISAPRE & 38 & 25,7 \\
\hline FONASA & 91 & 61,5 \\
\hline Otro & 8 & 5,4 \\
\hline No tiene & 11 & 7,4 \\
\hline \multicolumn{3}{|l|}{ Vivienda } \\
\hline Propia & 103 & 69,6 \\
\hline Arrendada & 24 & 16,2 \\
\hline Allegada & 21 & 14,2 \\
\hline \multicolumn{3}{|c|}{ Percepción del nivel de ingresos económicos familiar } \\
\hline Malo & 19 & 12,8 \\
\hline Aceptable & 116 & 78,4 \\
\hline Bueno & 13 & 8,8 \\
\hline
\end{tabular}

Fuente: "Conductas de autocuidado y manifestaciones de la mujer en su etapa perimenopausica, Comuna de Concepción, 2002".

Si se evalúa la presencia de hijos en hogar, se recoge que $84,5 \%$ de las mujeres tienen hijos viviendo en el hogar, quefluctúan entre 1 a 4 , losque pueden estar estudiando, trabajando o con alguna situación particular (de salud entreotras), por laqueno han dejado el hogar.
Esto es similar a lo encontrado en el Censo 2002, en cuyas estadísticas seencuentra quelas mujeres pueden tener hasta 10 hijos viviendo a su lado, si bien los motivos no seespecifican.

En materia laboral, un $53,4 \%$ señaló trabajar fuera del hogar, en forma remunerada, 
sin existir un grupo con fuerte tendencia respecto del tiempo quellevan laborando. El resto de las mujeres trabaja dentro de su hogar, lo que en el Censo 2002 clasificaron como quehaceres del hogar dentro de la población femenina no económicamente activa. Según esas estadísticas a nivel nacional, las mujeres que se desempeñan en quehaceres del hogar, están en un número mayor que las que desarrollan actividad remunerativa y que se encuentran clasificadas como población económicamenteactiva, en el área urbana, entrelos 45 y 54 años. Al consultarles cómo consideran el nivel de ingresos económicos de su familia, la respuesta mayoritaria es aceptable. En el grupo delas mujeres que desarrolla que haceres del hogar se reconoce que también dentro del mismo realizan labores que ayudan al sustento de su familia, aunque no se logra precisar porcentajes.

Con relación a su orientación religiosa, la mayor parte de las mujeres encuestadas refi- rieron ser católicas (73\%), dato homologable a lo recogido a través del Censo 2002 a nivel regional y nacional (no existe el dato a nivel de comuna), y lo mismo ocurre con las otras categorías; sin embargo, si bien un $73,6 \%$ del total de encuestadas practica su religión con variablefrecuencia, la mayoría delas quepractican son de orientación religiosa distinta a la católica. Cuando se les pregunta si pertenece a algún otro grupo dela comunidad, un $86,5 \%$ refiere que no lo hace.

Respecto a la previsión de salud, un $61,5 \%$ responde que pertenece a FONASA, frente a un $25,7 \%$ quepertenecealSAPRE. No existen datos del Censo 2002 que puedan corroborar esta información. Las mujeres al ser consultadas respecto del tipo de vivienda que habitan, refieren en un $69,6 \%$ que ésta es propia.

Al ser consultadas las mujeres respecto de su asistencia a controles de salud, un $77 \%$ señalan que lo hacen, siendo las causas variadas; las de mayor frecuencia son aquéllas de

Tabla 2. Características relacionadas con salud de las mujeres en etapa perimenopáusica. Comuna de Concepción, 2002.

\begin{tabular}{|l|r|r|}
\hline \multicolumn{1}{|c|}{ Variable } & $\mathrm{n}$ & $\%$ \\
\hline Asistencia a controles de salud & 114 & 77 \\
\hline SI & 34 & 23 \\
\hline NO & 63 & 42,6 \\
\hline Razones por las que asiste a controles de salud & 48 & 32,4 \\
\hline Preventivo & 12 & 8,1 \\
\hline Por enfermedad & 25 & 16,9 \\
\hline Otras razones & & \\
\hline No especifica razones &
\end{tabular}

Fuente: Idem Tabla 1.

tipo preventivo y por presencia de enfermedades. Entre otras razones señalan: presentar enfermedades en forma esporádica, o lo que denominan un "chequeo" por alguna molestia en particular. Cuando se les pregunta específicamente el tipo de asistencia de salud quesolicitan, un 40,9\% señala que visita profesionales de salud en forma particular y sólo un $39,9 \%$ acude el consultorio al que pertenece, lo que llama particularmente la atención si consideramos que, según sus respuestas al ítem previsión de salud, un gran porcentaje respondió que pertenecía a FONASA.

Respecto a la frecuencia con que asisten a controles desalud, un 38,5\% respondequelo hacen cada seis meses, principalmente las 
mujeres que presentan historia de enfermedades crónicas (como hipertensión, diabetes u otros problemas endocrinos, así como la presencia de algún quiste o tumor). No obstante, la diferencia con las mujeres que asisten en forma anual a controles de salud, es pequeña $(41,2 \%)$, cuyos motivos son similares a los mencionados anteriormente.

El tipo de profesional que las controla es principalmente el médico $(43,2 \%)$, seguido por matrona. Los profesionales de enfermería son los menos visitados (2,7\%).

Tabla 3. Características relacionadas con el período climatérico de las mujeres en etapa perimenopáusica. Comuna de Concepción, 2002.

\begin{tabular}{|c|c|c|}
\hline Variable & $\mathrm{n}$ & $\%$ \\
\hline \multicolumn{3}{|c|}{ Presencia de ciclos menstruales } \\
\hline $\mathrm{SI}$ & 80 & 54,1 \\
\hline NO & 68 & 45,9 \\
\hline \multicolumn{3}{|c|}{ Fecha de la última regla (FUR) } \\
\hline Menor a seis meses & 78 & 52,7 \\
\hline Mayor a seis meses & 70 & 47,3 \\
\hline \multicolumn{3}{|c|}{ Usuaria de Terapia Reposición Hormonal (TRH) } \\
\hline $\mathrm{SI}$ & 55 & 37,2 \\
\hline NO & 93 & 62,8 \\
\hline \multicolumn{3}{|c|}{ Sensación de beneficio manifestada por usuarias de TRH(de los 55) } \\
\hline $\mathrm{SI}$ & 45 & 85,5 \\
\hline NO & 8 & 5,4 \\
\hline
\end{tabular}

Fuente: Idem Tabla 1.

Las mujeres encuestadas responden afirmativamentea la pregunta sobre presencia de ciclos menstruales en un $54,1 \%$, señalando sólo un $38,5 \%$ que aún son ciclos regulares. Pero la pregunta respecto a la fecha dela última regla (FUR) ratifica la primera, ya que un $52,7 \%$ responde que la ha presentado dentro de los últimos seis meses. Se puede concluir que en esta muestra de mujeres, que poseen una media de edad de 50 años, la mitad de ellas está transitando por el periodo perimenopáusico. Este dato coincide con lo encontrado por Sanhueza, Valenzuela y Jara (1999) en Concepción, en que la edad promedio en quese presentó la menopausia fuede 50 años, fluctuando entre los 46 y 55 años. Un 37,2\% deellas refiereser usuaria deTRH (terapia de reposición hormonal), señalando un 85,5\% de las mujeres que responde afirmativamente, sensación de beneficio. 
Tabla 4. M anifestaciones del período perimenopáusico de la mujer.

\begin{tabular}{|c|c|c|c|c|c|c|}
\hline \multicolumn{2}{|c|}{ Indice } & Subíndice & $\mathrm{n}$ & $\%$ & Media & DS \\
\hline Vasomotor & Alfa: 7922 & $\begin{array}{l}\text { Bochornos } \\
\text { Transpiraciones nocturnas } \\
\text { Más transpiración de lo normal }\end{array}$ & $\begin{array}{l}57 \\
46 \\
38\end{array}$ & $\begin{array}{l}38,5 \\
31,1 \\
25,7\end{array}$ & $\begin{array}{l}1.47 \\
1.23 \\
1.00\end{array}$ & $\begin{array}{l}2.08 \\
2.03 \\
1.85\end{array}$ \\
\hline Psicosocial & Alfa: 7940 & $\begin{array}{l}\text { Descontento con la vida personal } \\
\text { N erviosismo, ansiedad } \\
\text { Pérdida de la memoria } \\
\text { H acer menos cosas de las acostumbradas } \\
\text { Sentirse deprimida o triste } \\
\text { Impaciencia con otras personas } \\
\text { Sentir necesidad de estar sola }\end{array}$ & $\begin{array}{l}37 \\
95 \\
72 \\
65 \\
82 \\
85 \\
78\end{array}$ & $\begin{array}{l}25,0 \\
64,2 \\
48,6 \\
43,9 \\
55,4 \\
57,4 \\
52,7\end{array}$ & $\begin{array}{l}0.95 \\
2.68 \\
1.53 \\
1.61 \\
2.16 \\
2.18 \\
1,98\end{array}$ & $\begin{array}{l}1.80 \\
2.38 \\
1.87 \\
2.03 \\
2.24 \\
2.28 \\
2.21\end{array}$ \\
\hline Física & Alfa: .8507 & $\begin{array}{l}\text { Flatulencia, gases o hinchazón abdominal } \\
\text { Dolores musculares o articulares } \\
\text { Sentirse cansada o agotada físicamente } \\
\text { Dificultad para dormir } \\
\text { Dolores de cabeza o nuca } \\
\text { Disminución de la fuerza física } \\
\text { Disminución de la resistencia física } \\
\text { Sensación de falta energía } \\
\text { Sequedad de la piel } \\
\text { Aumento de peso } \\
\text { Aumento de aparición de vellos en la cara } \\
\text { Aumento en la aparición de arrugas o } \\
\text { manchas } \\
\text { Retención de líquido } \\
\text { Dolor de cintura } \\
\text { Orina frecuentemente } \\
\text { Se orina al reírse, toser o hacer esfuerzo }\end{array}$ & $\begin{array}{r}108 \\
111 \\
94 \\
59 \\
88 \\
92 \\
95 \\
95 \\
84 \\
81 \\
22 \\
\\
86 \\
54 \\
69 \\
77 \\
51\end{array}$ & $\begin{array}{l}73 \\
75 \\
62,5 \\
39,9 \\
59,5 \\
61,5 \\
64,2 \\
64,2 \\
56,8 \\
54,7 \\
14,9 \\
58,1 \\
36,5 \\
46,6 \\
52 \\
34,5\end{array}$ & $\begin{array}{l}2.75 \\
3.11 \\
2.56 \\
1.63 \\
2.19 \\
2.25 \\
2.32 \\
2.34 \\
2.17 \\
2.29 \\
0.41 \\
\\
\\
\end{array}$ & $\begin{array}{l}21.5 \\
2.25 \\
2.31 \\
2.24 \\
2.22 \\
2.10 \\
2.05 \\
2.11 \\
2.20 \\
2.43 \\
1.10 \\
\\
1.91 \\
1.85 \\
2.13 \\
2.31 \\
1.97\end{array}$ \\
\hline Sexual & Alfa: .7317 & $\begin{array}{l}\text { Cambios en su deseo sexual } \\
\text { Sequedad vaginal durante el acto sexual } \\
\text { Evita las relaciones sexuales }\end{array}$ & $\begin{array}{l}63 \\
51 \\
71\end{array}$ & $\begin{array}{l}42,6 \\
34,5 \\
48\end{array}$ & $\begin{array}{l}1.75 \\
1.35 \\
1.82\end{array}$ & $\begin{array}{l}2.26 \\
2.03 \\
2.12\end{array}$ \\
\hline
\end{tabular}

Fuente: Idem Tabla 1.

Cabe señalar que para los valores de las medias y DS aquí señalados, se extrajeron de los rangos de la intensidad de las manifestaciones, considerando queéstas son escalas tipo Likert.

Al analizar cada una de las manifestaciones que son mencionadas como del período perimenopáusico, y se analiza por grupo de manifestación, encontramosquedentro delas vasomotoras no existe al guna que se presente frecuentemente, excepto los bochornos, los cuales no alcanzan a representar un $40 \%$ de la muestra.

Dentro de las manifestaciones psicológicas, lo más sobresalientees la presencia denerviosismo y ansiedad, quelos presentaban más del $50 \%$ de las mujeres.

La diferencia la marcan las manifestacio- 
nesfísicas citadas por las mujeres encuestadas, sobresaliendo la presencia de flatulencia, gases o hinchazón abdominal, los dolores musculares y/o articulares, orinar frecuentemente, el cansancio, disminución de la fuerza y de la resistencia física. Otros también mencionados, pero en menor cuantía, fueel dolor deca- beza, sequedad de la piel, aumento de peso y cambios en la piel, ya sean manchas o arrugas.

Los aspectos sexuales, si bien no sobrepasan el 50\%, tampoco dejan de tener importancia los cambios que experimentan las mujeres en el deseo sexual, junto con evitar las relaciones sexuales.

Tabla 5. Actividades de autocuidado durante el período perimenopáusico.

\begin{tabular}{|c|c|c|c|c|c|c|}
\hline \multicolumn{2}{|l|}{ Indice } & Subíndice & $\mathrm{F}$ & $\%$ & Media & DS \\
\hline $\begin{array}{l}\text { Actividades } \\
\text { de apoyo al } \\
\text { funcionamiento } \\
\text { normal }\end{array}$ & Alfa: .6301 & $\begin{array}{l}\text { Aceptación de la etapa } \\
\text { Autovaloración } \\
\text { Asistencia a controles de salud } \\
\text { Realizar más actividad física } \\
\text { Autoaprendizaje } \\
\text { Realización de actividades de distracción } \\
\text { Búsqueda de apoyo } \\
\text { Búsqueda de consejos } \\
\text { Confianza en otros }\end{array}$ & $\begin{array}{r}128 \\
79 \\
119 \\
90 \\
127 \\
44 \\
106 \\
98 \\
95\end{array}$ & $\begin{array}{l}93,2 \\
53,4 \\
80,4 \\
60,8 \\
85,8 \\
29,7 \\
71,6 \\
66,2 \\
64,2\end{array}$ & $\begin{array}{l}4.22 \\
2.28 \\
2.28 \\
2.70 \\
3.72 \\
1.88 \\
2.96 \\
2.85 \\
2.73\end{array}$ & $\begin{array}{l}1.16 \\
1.47 \\
1.58 \\
1.58 \\
1.42 \\
1.52 \\
1.52 \\
1.60 \\
1.57\end{array}$ \\
\hline $\begin{array}{l}\text { Actividades de } \\
\text { prevención o } \\
\text { control de los } \\
\text { procesos de } \\
\text { enfermedad o } \\
\text { lesión }\end{array}$ & Alfa: .5879 & $\begin{array}{l}\text { Preocupación por la etapa } \\
\text { Ingesta de vitaminas } \\
\text { Búsqueda de ayuda terapéutica } \\
\text { Registro de actividades } \\
\text { Actividad física } \\
\text { Distracciones sociales }\end{array}$ & $\begin{array}{r}123 \\
63 \\
51 \\
44 \\
81 \\
59\end{array}$ & $\begin{array}{l}83,1 \\
42,6 \\
34,5 \\
29,7 \\
54,7 \\
39,9\end{array}$ & $\begin{array}{l}3.42 \\
2.01 \\
1.82 \\
1.89 \\
2.57 \\
1.93\end{array}$ & $\begin{array}{l}1.53 \\
1.44 \\
1.30 \\
1.49 \\
1.67 \\
1.35\end{array}$ \\
\hline $\begin{array}{l}\text { Actividades de } \\
\text { promoción del } \\
\text { bienestar }\end{array}$ & Alfa: .6712 & $\begin{array}{l}\text { Preocupación por su aspecto físico } \\
\text { Búsqueda de alternativa de solución } \\
\text { Fe } \\
\text { Búsqueda de soluciones } \\
\text { Aceptación de cambios corporales } \\
\text { Cuidado de sí misma } \\
\text { Ocupaciones extralaborales } \\
\text { M ejora su dieta } \\
\text { Realiza actividades saludables } \\
\text { Planifica sus actividades } \\
\text { Evita situaciones difíciles } \\
\text { Realiza labores domésticas } \\
\text { Disminuye carga de trabajo } \\
\text { Participa en grupos de apoyo } \\
\text { Busca información respecto al tema }\end{array}$ & $\begin{array}{r}139 \\
112 \\
124 \\
114 \\
121 \\
137 \\
131 \\
113 \\
107 \\
106 \\
96 \\
111 \\
138 \\
125 \\
125\end{array}$ & $\begin{array}{l}95,9 \\
75,7 \\
83,8 \\
77 \\
81,8 \\
92,6 \\
88,5 \\
76,4 \\
72,3 \\
71,6 \\
64,9 \\
75 \\
93,2 \\
84,5 \\
84,5\end{array}$ & $\begin{array}{l}3.95 \\
3.29 \\
3.47 \\
3.30 \\
3.22 \\
4.20 \\
3.80 \\
3.61 \\
3.41 \\
2.89 \\
3.09 \\
2.97 \\
3.28 \\
3.02 \\
3.76\end{array}$ & $\begin{array}{l}1.26 \\
1.59 \\
1.48 \\
1.59 \\
1.47 \\
1.23 \\
1.41 \\
1.68 \\
1.52 \\
1.52 \\
1.62 \\
1.65 \\
1.57 \\
1.52 \\
1.45\end{array}$ \\
\hline
\end{tabular}

Fuente: Idem Tabla 1. 
Las actividades de autocuidado que realizan las mujeres en etapa perimenopáusica se han dividido en tres grupos, de los cuales en aquellas actividades de apoyo al funcionamiento normal y aquellas actividades destinadas a la prevención o control de procesos deenfermedad tienen los alfa más bajos, pese a lo cual demuestran congruencia entre sus ítems.

Dentro de las actividades de autocuidado que las mujeres realizan, destacan las actividades de promoción debienestar, sobresaliendo entre ellas la preocupación por su aspecto físico, el cuidado de sí misma, las ocupaciones extralaborales y la disminución de la carga laboral. Resulta contradictorio este resultado, ya que ellas responden afirmativamente cuando se les consulta si participan en grupos deapoyo como actividad deautocuidado, comparando con la respuesta a la pregunta sobre pertenencia a un grupo de la comunidad, en donde la mayoría de las mujeres respondió negativamente. Otra respuesta interesante es la de buscar información respecto al tema, algo que, como se verá en la discusión, es una propuesta permanenteen los pocos estudios real izados respecto a esta materia.

O bservando la tabla, se puede ver que dentro de las actividades de apoyo al funcionamiento normal, las menos utilizadas son las actividades de distracción, en comparación con las más utilizadas como son las actividades de búsqueda de apoyo y consejos; el resto de las actividades dentro de este grupo tiene frecuencia dispersa. En este grupo existe disparidad en algunosítemes cuando responden las mujeres a la frecuencia con que realizarían determinadas actividades, como la aceptación dela etapa, el autoaprendizajey la realización de actividades de distracción.

Si se analiza las actividades de apoyo a la prevención o control de procesos deenfermedad o lesión, las frecuencias también son dispersas. Las actividades menos realizadas son la ingesta de vitaminas, el registrar sus actividades y las distracciones sociales. Aquí la pre- ocupación por la etapa es el ítem quemás disparidad presenta respecto de las otras actividades para prevenir procesos de enfermedad o lesión.

\section{DISCUSIÓN Y COMENTARIOS}

La etapa denominada perimenopausia es una etapa normal del desarrollo de la mujer, necesaria de seguir profundizando en ella por los integrantes del equipo de salud, dado que en variadas ocasiones se toman decisiones influidas por conceptos estereotipados deesta etapa, ajenos a las evidencias disponibles y creyendo que las mujeres presentan, sino todas, al menos algunas de las manifestaciones "típicas", las que también se han transformado en "características deeste período". Si bien este estudio presenta falencias (como el no haber sido aplicado en zona rural, por ejemplo), se presenta descripción bastante acabada de variables sociales, bio-demográficas y algunas características relacionadas con salud respecto de las mujeres que vivencian el período perimenopáusico, para dar a conocer su realidad, sin circunscribirse a una institución específica, sino que en su contexto habitual.

Dentro de las actividades de autocuidado más comunes entrelas mujeres perimenopáusicas están aquellas tendientes a la promoción del bienestar. Pudiera ser que la razón de llevar a cabo estetipo deactividades tenga como finalidad intentar comprender mejor la etapa quevivencian, ya que destacan principalmente la búsqueda de apoyo, información y consejo de otros. La relevancia que este aspecto tiene para enfermería es que se identifica la necesidad deconformar grupos deapoyo para esta etapa, requiriéndose para expresar distintostópicos que aclaren las interrogantes de las mujeres, teniendo en cuenta quelas mujeres además en esta etapa pueden estar atravesando por otros problemas de salud que se entremezclan con susnecesidadesactuales. En este punto es donde también es prioritario 
desarrollar objetivos en el área de asistencia primaria, para dar una atención centrada en la real problemática de las mujeres en etapa dela perimenopausia, evitando, en cuanto esté de parte de quienes formulen estos programas, dificultades de acceso a la información, como las hallara Domm (2000) en Estados Unidos. Es coincidente este hallazgo con la demanda demayor información quehicieron las mujeres en el estudio de Pina (1997), en una localidad española.

Otro aspecto a abordar, y no menos importante que el anterior, dice relación con la preocupación por su aspecto físico, el cuidado desí misma, las ocupaciones extralaborales y la disminución de la carga laboral, todas actividades de promoción del bienestar; los datos precedentes se relacionan directamente con aquellos estudios que evidencian que las mujeres presentan una dramática pérdida deautoestima, como el publicado por Blümel (2000), situación rel evante en el estudio citado dado quese trataban demujeres aún jóvenes, muchas de ellas en etapa de plena actividad y realización; en este trabajo las mujeres oscilaban entre 45 y 54 años de edad, período en que las mujeres trabajan, aún tienen hijos en el colegio y tienen que interactuar, en su mayoría, con muchas personas, algunas de ellas, de hecho, recién se incorporan o reincorporan en este período a la fuerza laboral.

Se reconoce en este estudio que no todas las mujeresque están transitando por esta etapa presentan manifestaciones y aquéllas que aquí son mencionadas por las mujeres no coinciden con la literatura revisada. En la mayoría de los casos las mujeres de este estudio consultaban a los profesionales dela salud por otros motivos ajenos a la etapa. Dentro de las manifestaciones por las que consultaban, tampoco hay coincidencia entre estudios, ya que en las mujeres de la Comuna de Concepción sus consultas tienen quever en su mayoría con el ámbito físico: flatulencia, gases o hinchazón abdominal, dolores musculares y articulares, sentirse cansada, disminución de la re- sistencia física y sensación de falta de energía y con el ámbito psicosocial: nerviosismo, ansiedad, impaciencia con otras personas. En cambio, en literatura las consultas se refieren a manifestaciones de origen vasomotor y sexuales, como lo señala Blümel en 1991. Y las manifestaciones menos citadas son las de orden vasomotor y sexual, que han llegado a ser base de algunos argumentos por los que se utiliza la Terapia de Reposición H ormonal.

Por ende, se colige que este período es necesario enfrentarlo, desde un punto de vista sanitario y social, con una programación acordey con la naturalidad y tranquilidad con que se enfrenta la etapa reproductiva u otras dela vida de la mujer, pero contando con programas e infraestructura acordes a las necesidades expresadas por las mujeres en su contexto habitual. Tal vez sería apropiado considerar la necesidad de crear un programa de salud específico para la mujer en período perimenopáusico, unido a los demás programas existentes para otros problemas de salud, considerando que este período posee características específicas que deben ser abordadas. Enfermería en este aspecto puede proporcionar tanto herramientas de apoyo al bienestar como educativas, que ayuden a las mujeres a enfrentar esteperíodo, haciendo presenteque el grupo menos consultado por las mujeres son estos profesionales, lo cual representa un desafío de participar activamentetambién en esta etapa. Se reconoce la existencia de profesionales de enfermería especialistas en salud mental y sexualidad humana, que pueden y deben prestar la ayuda terapéutica específica para las necesidades de las mujeres en este período.

\section{REFERENCIASBIBLIOGRÁFICAS}

AVIS, N. E., Stellato, R., Crawford, S., Johannes, C., Longcope, C. (2000). Is there an association between menopause status and sexual functioning? M enopause, vol. 7; 5; pp. 297-309.

BLÜ M EL, J. E., Binfa, I., Framegna,G., Tacla, X., 
Aracena, B., Cumsille, M . A. (1998). Efecto del climaterio en la calidad de vida. Rev. Chi. Obst. Ginecol. Santiago, 63 (6): 437-445.

BLÜ M EL, J., Brandt, A., Tacla, X. (1991). La menopausia: ¿Una enfermedad? Rev. Chi. O bstet. Ginecol., Santiago N ${ }^{\circ} 56$, pp. 268-273.

BLÜM EL, J., Castelo-Branco C., Binfa, L., y cols. (2000). Quality of life after the menopause: a population study. Maturitas, 15, 34 (1): 17-23.

BOGGS, P.; Rosenthal, M. (2000). Helping Women Help Themselves: Developing a Menopause Discussion Group. Clin. Obst. And Gynecol., vol. 43. $N^{\circ} 1$, pp. 207-212.

CRISOSTO, C.: IN J. Blümel (Ed) (1993). Menopausia: Orientaciones diagnósticas y terapéutica. Santiago, Chile: Visual Ediciones.

DOM M , J. Factors affecting access to menopause information (2000). M enopause. vol. 7; 1; pp. 62-67.

DOS Reis, A. (1999). Envejecimiento femenino y menopausia: un abordaje antropológico. Cuadernos mujer salud, El Caribe, pp. 40-44.

FLINT, M . (1975). The menopause: reward or publishment. Psycosomatics, vol. 16, N 4, pp. 161-163.

GERER, G. (1993). El cambio, mujeres, vejez y menopausia. España, Ed. Ana Grama.

GON ZÁLEZ, O., Arteaga, E., Contreras, P. (1998). Menopausia y patologías asociadas: prevención y tratamiento. Santiago, Chile. Ed. Sociedad Chilena de Climaterio.

GREEN DALE, G. \& Judd, H . (1993). The menopause: health implications and clinical management). JAGS, 41: pp. 426-436.

GUTIÉRREZ, M ., Bas, P. (2001). M enopausia en atención primaria. Atención Primaria. España.

HERNÁN DEZ, Fernán dez y Baptista (1998). M etodología de la investigación. 2ª edición, Edit. Mc GrawHill Interamericana S.A. M éxico.

HILDITCH, J. R., Lewis, J., Peter, A., Vanmaris, B., Ross, A., Franssen E., Gordon, G., Norton, P., Dunn, E. (1996). A menopause-specific quality of life questionnaire: development and psychometric properties. M aturitas, 2: 161-175.

IBARRA, L., Diez García, M., Ruiz, T., Coronado, R., Pacheco, R. (2001). Factores biopsicosociales de la rehabilitación durante la menopausia. Rev. M exicana de M edicina Física y Rehabilitación; 13: 5-8.

LAVÍN, P. (1998). Envejecimiento poblacional y menopausia. In: González y col. M enopausia y patologías asociadas. Prevención y tratamiento. Santiago, Chile, Ed. Sociedad Chilena del Climaterio.

LI, S., H olm, K., Gulanick, M . y cols. (2000). Perimenopause and the Quality of Life. Clinical Nursing Research, vol. 9, $N^{\circ} 1$, pp. 6-26.
LOCK, M . (1988). N ew japanesemythologies: faltering discipline and the ailing housewife. American Ethnologist, vol. 15, N 1 , pp. 42-61.

M AM EDE, M ., De Souza, L. y cols. (1992). M enarcaMenopausa: quando ocorrem? Jornal Brasileiro de Ginecología. Brasil. V.102, N os 11 y 12, pp. 441-444.

M C KIN LAY y cols. In: Hyde, J. (1995). Psicología de la mujer. La otra mitad de la experiencia humana. M adrid, España, Ed. M orata, 509 pp.

M EDINA, E., Arteaga, P. y cols. (1990). Algunos efectos del tabaquismo en la mujer. Rev. M éd. DeChile, Santiago, v. 118, N 03 , pp. 253-258.

MERCK, Laboratorios (2001). ¿Qué es la perimenopausia? Rev. Galenus, Lab. M erck, año 13, № 50, Santiago, Chile, agosto.

NAVARRO, D., Fontaine, Y. (2001). Síndrome climaté rico: su repercusión social en mujeres de edad me diana. Rev. Cubana M ed. Gen. Integr.; 17(2): 16976.

NICO L-SM ITH , L. (1996). Causality, menopause, and depression. A critical review of theliterature. BMJ, vol. 313, N 12 , pp. 29-32.

OLÁZABAL, J. C., García, R., M ontero, J., García, J. F., Pastor, F. (2000). La atención a la mujer menopáusica: un objetivo a desarrollar desde la atención primaria. Atención primaria. 0 ct., vol. 26, № 6, pp. 405-414.

OREM , D. (1993). Consideraciones sobre la salud, los cuidados de la salud y la Enfermería. In: M odelo de Orem: conceptos de enfermería en la práctica. Pennsylvania, Masson y Salvat, 421 pp.

OREM , D. (1980). N ursin Concepts of Practice. Second Edition, M ac Graw-Hill Book Company. New York, $232 \mathrm{pp}$.

OSBORN, M . (1984). Depression at the menopause. British Journal of Hospital Medicine.

PAPALIA, D.; Wendkos, S. (1992). Desarrollo humano. Edit. Mc Graw-Hill InteramericanaS.A., Santefé de Bogotá, Colombia.

PARRA In: González, O., Arteaga, E., Contreras, P. (1998). M enopausia y patologías asociadas: Prevención y tratamiento. Santiago, Chile. Ed. Sociedad Chilena de Climaterio.

PINA, CARRASCO y cols. (1997). H ábitos de salud en la mujer menopáusica: un estudio sobre sus necesidades reales. Enfermería Clínica, vol. 7, N 4, pp. 175-180.

PROGRAM A DE SALUD DE LA MUJER. MINISTERIO DE SALUD, CHILE, 1995.

REBAR, R. et al. (2000). Clinical challenges of Perimenopause: consensus opinion of the North American Menopause Society. M enopause, vol. 7; 5; pp. 5-13. 
SAN HUEZA, O., Valenzuela, S.; Jara, P. (1997). M enarquia y menopausia, desde una perspectiva de mujeres (artículo no publicado).

SANTOS, A., Sanhueza, O. (2000). Comentary article "Perimenopause and the Quality of Life". Clinical Nursing Research, vol. 9 n. 1, 6-26.

SARDEN BERG, C. M. B. (1994). De sangrías, tabus e poderes: a menstruacao numa perspectiva sócioantropológica. Estudos Feministas, vol. 2, N² 2, pp. 315-345.

SERNAM , Documento de trabajo N 24 (1994). Perfil demortalidad y epidemiológico dela mujer. Servicio Nacional de la Mujer, Santiago, Chile.

SH ALASTER, In Pina. y cols. (1997). H ábitos de salud en la mujer menopáusica: un estudio sobre sus necesidades reales. Enfermería Clínica, vol. 7, $N^{\circ} 4$, pp. 175-180.

The boston women health book collective (2000). Nuestros cuerpos, nuestras vidas. Barcelona, España, Plaza Janes Editores S.A., 638 pp.
UPJOHN In: Papalia. D.; Wendkos, S. (1992). Desarrollo humano. Edit. M c Graw-Hill Interamericana S.A. Santefé de Bogotá, Colombia.

VALDIVIA, G. (1993). Aspectos clínico epidemiológicos del climaterio y menopausia. Boletín Esc. de Medicina. PUC, v. 22, $N^{\circ} 1$, pp. 7-8.

VALENZUELA, S., Paravic., T., Sanhueza, O. y cols. (2001). Programa de salud de la mujer en Chile: Relación entrelo teórico y lo empírico en la comuna de Concepción, en prensa, Ciencia y Enfermería, Revista I beroamericana de Investigación.

VALLS C. (1999). Cada vez vivimos más pero... ¿vivimos mejor? Cuadernos mujer salud, El Caribe, pp. 40-44.

VODA, Ann (1988). M enopausia. Manual para mujeres. In EPAS. Educación para el autocuidado en salud. Vol. V, № 4, julio 1988. Pontificia Universidad Católica de Chile, Santiago, Chile.

WERTHEIN , S. (1999). Abanicos: de las paradojas a la madurez. Cuadernos mujer salud, El Caribe, 1999, pp. 40-44. 